\title{
Projektstudie zum Logistik- und Technologiekonzept für eine Drehautomatenfertigung
}

\author{
Robert Deininger
}

\section{Einleitung}

Im Rahmen der Lehrveranstaltung Produktionslogistik des Studienganges Unternehmenslogistik erhielt unser Projektteam den Auftrag zur Entwicklung eines Technologie- und Logistikkonzeptes für die Montage von Drehautomaten mit integrierter Farbgebung und Verpackung einschließlich der Erstellung der Konzeption für die Montagehalle.

Das Projektteam bestand aus 5 Studenten des Studienganges Logistik der Technischen Fachhochschule Wildau. Die Projektarbeit wurde durch Herrn Robert Deininger geleitet. Die Systemanalyse sowie die Prozessbeschreibung wurde unter Verantwortung von Herrn Sebastian Forkert erstellt. Das Hallenlayout und die Erfassung der Basisdaten erfolgte unter Leitung von Herrn Dirk Ryborz. Die weiteren Teammitglieder Frau Silvana Adolph und Herr Jan-Jaap van Wikselaar wurden bedarfsweise in die Bearbeitung einzelner Aufgaben eingebunden. Die Betreuung des Projekts erfolgte durch den Dozenten der TFH Wildau, Herrn Prof. Dr.-Ing. habil. Bernd Hentschel.

Als wesentliche Ausgangsdaten waren die Hallenabmessungen sowie die Takte der einzelnen Montageschritte gegeben. Ferner standen folgende Ausgangsprämissen zur Verfügung:

- Anzahl der Tore für die Anlieferung von Baugruppen

- Anzahl der technologischen Stationen der Montage

- Anzahl der Arbeitskräfte pro Montagetakt

- Montagezeiten je Arbeitsgang

- Erzeugnisspezifische Vorgaben für die einzelnen Baugruppen (Maße, Gewichte)

- Standzeiten für Grundierung und Lackierung sowie Trocknung der Baugruppen

Ferner sollte nach Möglichkeit auf Zwischenlagerstufen verzichtet und ein Anlieferkonzept für die Baugruppen auf Just-in-Time oder Ship-to-Line Basis erstellt werden.

\section{Technologischer und Logistischer Ablauf}

Der technologische Ablauf beschreibt die Verrichtungsfolge in der bestimmte Tätigkeiten auszuführen sind. Die Technologie bestimmt dabei die erreichbare Produktivität qualitativ als auch quantitativ. Unter Technologie versteht man damit die Gesamtheit der Verfahren zur Produktion und der zur Verfügung stehenden Mittel.

Der Bereich Logistik beschreibt alle Zwischenschritte der Fertigung. Damit umfasst die Logistik alle Aktivitäten im physischen Raum zur Zeitüberbrückung von Gütern und Personen, einschließlich deren Umgruppierung. Der technologisch-logistische Ablauf beschreibt damit wie eine Art Prozessbeschreibung alle Aktivitäten in deren Verrichtungsfolge mit deren Normzeit zur Verrichtung der Montage- und Handlingschritte. Zusätzlich wurden die Anzahl der notwenigen Arbeitskräfte für den jeweiligen Arbeitsschritt ermittelt.

Als Hilfsmittel standen Programme wie Microsoft Excel und Microsoft Projekt zur Verfügung. Zur Erstellung eines Gesamtkonzeptes wurden alle Teilabläufe einschließlich aller Teilschritte von der Anlieferung bis zur Endmontage zeitlich und räumlich zugeordnet.

Von der Baugruppenanlieferung bis zur Fertigstellung des Drehautomaten wurden so ca. 200 Schritte zeitlich und räumlich erfasst. Dies wird am Beispiel der Anlieferung der Baugruppen für ein Los von 3 Drehmaschinen-Unterkästen dargestellt (Abb.1).

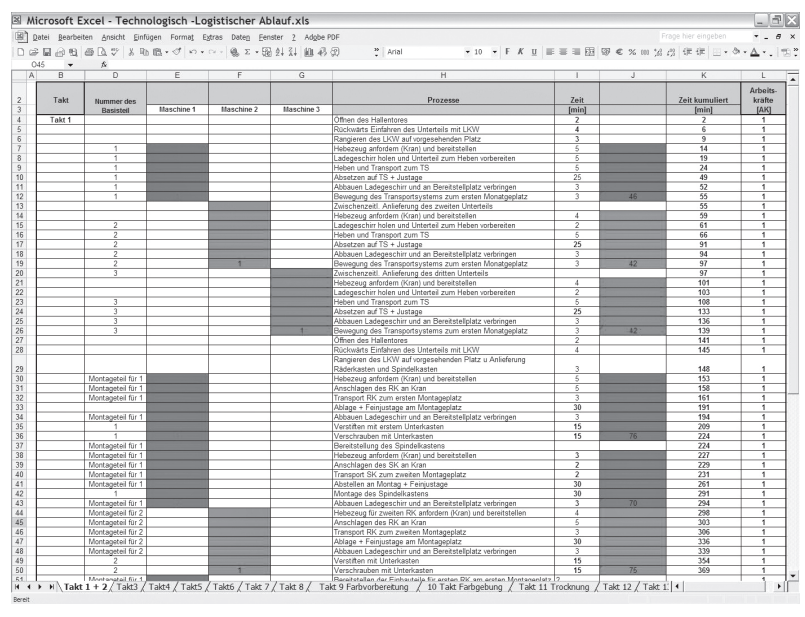

Abb. 1: Technologisch-logistischer Ablauf

In dieser Abbildung sind die einzelnen technologischen Schritte ihrer Ausführung nach sowie die Abfolge der logistischen Abläufe dargestellt. Analog wurde diese Analyse für alle übrigen Teilschritte des Produktionsprozesses betrachtet. Durch diese Prozessbeschreibung ist für jeden Mitarbeiter erkennbar, welche Schritte er wann, in welcher Zeit und an welchem Ort zu erfüllen hat. Für den Mitarbeiter ist damit auch erkennbar, welchen Teil er im Gesamtprozess darstellt und welche Schritte ihm vor- und nachgelagert sind. Dadurch ist eine Standardisierung der Abläufe möglich. Die Prozesse werden transparent und sie können auf dieser Grundlage optimiert, verändert oder neu gestaltet werden. Durch die Prozessanalyse des technologisch-logistischen Ablaufes werden auch Probleme visualisiert und können mit einem systematischen Problemlösungsverfahren untersucht werden. Dabei wurden folgende Grundsätze für die Lösung angewandt.

1 Problem analysieren

2 Ursache- Wirkungsprinzip anwenden, d. h. alle Einflüsse auf das Problem erfassen

3 Maßnahmen für die Lösung generieren und anwenden

4 Erfolg der Problemlösung prüfen 
Der technologisch-logistische Ablauf stellt damit die Gesamtheit aller theoretischen Untersuchungen zum Prozess der Drehautomatenfertigung dar und ist die Grundlage der weiteren Arbeit für die Produktionsplanung.

\section{Lösungskonzept}

Die Betrachtung einer möglichen Lösung erfolgte durch Variantenanalyse mit der die Kenngrößen Platzbedarf, Rüstzeit, Standzeit und Transportgeschwindigkeit verglichen wurden.

Die Variantenanalyse erfolgte dabei auf der Grundlage der gegebenen Fertigungstakte:

\begin{tabular}{|l|l|}
\hline Takt 1 & $\begin{array}{l}\text { Anlieferung des Unterkastens im Wareneingang. } \\
\text { Sichtkontrolle, mechanische Kontrolle, Dichtheitskontrolle } \\
\text { (Ultraschall). }\end{array}$ \\
\cline { 2 - 2 } & $\begin{array}{l}\text { Zwischenlagerung im Pufferlager bzw. Transport zum } \\
\text { 1. Montageplatz. Anlieferung Räderkasten, Spindelkasten, } \\
\text { Oberteil (Anlieferkonzept: Just-in-Time) }\end{array}$ \\
\cline { 2 - 2 } & Zeitansatz 60 min / 1 Arbeitskraft \\
\hline \multirow{5}{*}{ Takt 2 } & $\begin{array}{l}\text { Ausrichtung des Unterkastens in Waage-Justage auf dem } \\
\text { Transportsystem }\end{array}$ \\
\cline { 2 - 3 } & $\begin{array}{l}\text { Verbohren und Verschrauben des Räderkastens mit dem } \\
\text { Spindelkasten }\end{array}$ \\
\cline { 2 - 3 } & Zeitansatz 90 min / 1 Arbeitskraft \\
\hline Takt 3 3 & Einbau der Spindeltrommel und Laufprüfung \\
\cline { 2 - 3 } & Zeitansatz 300 min / 2 Arbeitskräfte \\
\cline { 2 - 3 } & Einbau des Antriebsmotors und der Drehzahlwechselräder \\
\cline { 2 - 3 } & Zeitansatz 240 min / 1 Arbeitskraft \\
\hline Takt 4 & $\begin{array}{l}\text { Montage von Mittelwelle, Hauptschlitten, Schneckenwelle } \\
\text { und oberer Steuerwelle einschließlich der Funktionsprüfung }\end{array}$ \\
\cline { 2 - 3 } & Zeitansatz 1170 min / 1 Arbeitskraft \\
\hline
\end{tabular}

\begin{tabular}{|c|c|}
\hline \multirow[t]{2}{*}{ Takt 5} & $\begin{array}{l}\text { Montage Kurvenscheibe für Oberschlitten und ggf. } \\
\text { Kurventrommel für unabhängige Vorschubeinrichtung }\end{array}$ \\
\hline & Zeitansatz $420 \mathrm{~min} / 1$ Arbeitskraft \\
\hline \multirow[t]{2}{*}{ Takt 6} & $\begin{array}{l}\text { Montage untere Steuerwelle, Schaltung für Spindeltrommel, } \\
\text { Kurvenscheiben für Unter- und Seitenschlitten, Schnecken- } \\
\text { welle für unteren Steuerwellenantrieb, Kurventrommel für } \\
\text { unabhängigen Vorschub }\end{array}$ \\
\hline & Zeitansatz 900 min / 2 Arbeitskraft \\
\hline \multirow[t]{4}{*}{ Takt 7} & Montage Pumpenkombination \\
\hline & Zeitansatz $180 \mathrm{~min} / 1 \mathrm{AK}$ \\
\hline & Ein und Testlauf des Drehautomaten \\
\hline & Zeitansatz $2280 \mathrm{~min} / 1$ Arbeitskraft \\
\hline \multirow[t]{2}{*}{ Takt 8} & $\begin{array}{l}\text { Transport des Drehautomaten mit Hilfe des } \\
\text { Transportsystems zur Farbvorbehandlung }\end{array}$ \\
\hline & Zeitansatz $180 \mathrm{~min} / 1$ Arbeitskraft \\
\hline \multirow[t]{2}{*}{ Takt 9} & $\begin{array}{l}\text { Farbvorbehandlung des Drehautomaten (Spachteln, } \\
\text { Schleifen und Abkleben) }\end{array}$ \\
\hline & Zeitansatz $360 \mathrm{~min} / 2$ Arbeitskräfte \\
\hline \multirow[t]{3}{*}{ Takt 10} & Antransport des Drehautomaten in die Farbgebung \\
\hline & Spritzen des Vorlack \\
\hline & Zeitansatz $270 \mathrm{~min} / 1$ Arbeitskraft \\
\hline \multirow[t]{2}{*}{ Takt 11} & Transport in die Trockenkabine und Trocknen \\
\hline & Zeitansatz $60 \mathrm{~min} / 1$ Arbeitskraft \\
\hline \multirow[t]{2}{*}{ Takt 12} & $\begin{array}{l}\text { Optische Prüfung und Transport Transport zum zweiten } \\
\text { Spritzen }\end{array}$ \\
\hline & Zeitansatz $60 \mathrm{~min} / 1$ Arbeitskraft \\
\hline \multirow[t]{2}{*}{ Takt 13} & $\begin{array}{l}\text { Einfahren des Drehautomaten in die Spritzkabine und } \\
\text { Spritzen des Decklack }\end{array}$ \\
\hline & Zeitansatz $60240 \mathrm{~min} / 1$ Arbeitskraft \\
\hline \multirow[t]{2}{*}{ Takt 14} & Transport in die Trockenkabine und Trocknen \\
\hline & Zeitansatz $60 \mathrm{~min} / 1$ Arbeitskraft \\
\hline \multirow[t]{3}{*}{ Takt 15} & Bereitstellung des Drehautomaten zur Verpackung \\
\hline & Verpackung des Drehautomaten \\
\hline & Zeitansatz $360 \mathrm{~min} / 2$ Arbeitskräfte \\
\hline \multirow[t]{2}{*}{ Takt 16} & Verladung der Maschinen \\
\hline & Zeitansatz 20 min / 1 Arbeitskraft \\
\hline
\end{tabular}

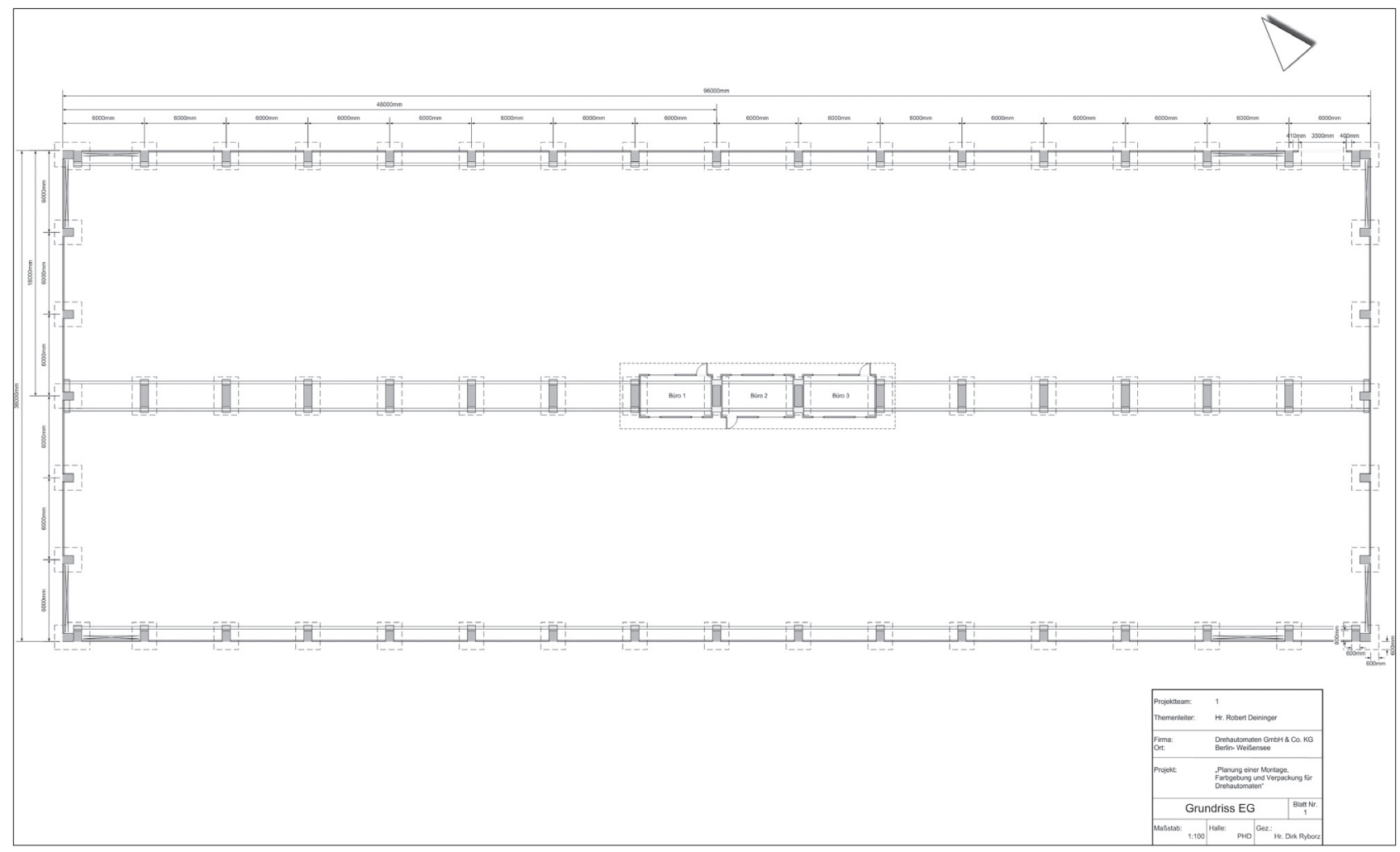

Abb. 2: Hallengrundriss 
Nach der Analyse der einzelnen Takte wurde mit der Planung des Hallen-Layouts auf der Grundlage der vorgegebenen Hallendaten begonnen. Für die Erstellung der Hallenskizzen standen die Programme AutoCAD und Mircosoft Viso zur Verfügung. Aufgrund von vorliegenden Studienkenntnissen und dem engen Zeitfenster wurde das System Microsoft Visio verwandt. Folgende Hauptparameter der zweischiffigen Halle waren dabei gegeben: Länge 96 $\mathrm{m}$, Breite $2 \times 18 \mathrm{~m}$ mit $6 \mathrm{~m}$ Stützenabstände in Längsrichtung. Den damit erstellten Hallengrundriss zeigt Abbildung 2. In den damit erstellten Hallenplan wurde zentral der Bürobereich und in der zweiten Ebene der Leitstand der Halle vorgesehen.

Da der Hallengrundriss die physische Grenze der Produktionskapazität bildet, wurden mehrere Varianten mit dem Ziel untersucht, auf der Grundlage der gegebenen Basisdaten und der gegebenen Mittel das Maximalprinzip durchzusetzen, d.h. das größtmögliche Ergebnis zu erreichen. Um den Logistikaufwand so gering wie nötig zu halten, wurde die Montage als Fließfertigung gewählt. Da die Qualität des Erzeugnisses von entscheidender Bedeutung ist, wurden die Grundsätze des Qualitätsmanagement konsequent berücksichtigt.

\subsection{Auswahl der Technologien}

Da die in der Fertigung befindlichen Maschinen bei Störungen nur mit extrem großem Handling-Aufwand aus der Montagelinie zu nehmen sind, wurde die Möglichkeit des Kraneinsatzes untersucht. Mit einem Brückenkran können Lasten in alle drei Achsrichtungen gleichzeitig bewegt werden. Das Arbeitsfeld des Kranes wird nur durch die Abmessungen von Laufkatze, Kranbrücke, Fahrwerke und Seilantrieb eingeschränkt. Die Ausrüstung der Halle mit Kranbrückenträgern ist möglich. Ein Kran ermöglicht auch das in Takt 1 beschriebene Umsetzen der angelieferten Großteile vom Lastkraftwagen auf das Transportsystem. Des weiteren ist es möglich am Montageplatz 1 mit Hilfe des Kranes ohne zusätzlichen Aufwand Montageteile wie z.B. den Spindelkasten am Unterkasten zu montieren. Durch weitere Untersuchungen der notwendigen Transport- und Umsetzungsanforderungen wurde der Einsatz von vier Brückenkransystemen mit je $32 \mathrm{t}$ Tragfähigkeit konzipiert.

Ein reibungsloses Handling innerhalb der Produktion erfordert ein Transportsystem, dass die Anforderungen der Produktion vollständig erfüllt.

In einer Variantenuntersuchung wurden die Leistungsparameter sowie Vor-und Nachteile der möglichen Transportsysteme verglichen. Auf dieser Grundlage wurde ein flurgebundenes Transportsystem als effizienteste Lösung ermittelt. Die weitere Auswahl wurde nach folgenden Kriterien vorgenommen:

\begin{tabular}{|l|l|}
\hline Fahrzeugbezogene & - Fahrantrieb \\
\cline { 2 - 2 } Auswahlkriterien & - Bauform \\
\cline { 2 - 3 } & - Lenksystem \\
\cline { 2 - 2 } & - Lenkart \\
\cline { 2 - 2 } & - Bedienart \\
\cline { 2 - 2 } & - Tragfähigkeit \\
\cline { 2 - 2 } & - Leistung \\
\hline & - Fahrgeschwindigkeit \\
\hline
\end{tabular}

\begin{tabular}{|l|l|}
\hline Einsatzbezogene & - Transportgut \\
\cline { 2 - 2 } Auswahlkriterien & - Einsatzbedingungen (Arbeitsumgebung) \\
\cline { 2 - 2 } & - Auslastung des Flurförderzeuges, Einsatzzeit \\
\hline
\end{tabular}

Vorschriftenbezogene - Unfallverhütungsvorschriften

\begin{tabular}{l|l} 
Auswahlkriterien & - Arbeitssicherheit
\end{tabular}

Unter Einbeziehung dieser Kriterien ist ein fahrerloses Transportsystem als Lösung ermittelt worden, welches mit automatisch gesteuerten Fahrzeugen arbeitet. Die Transportwagen bewegen sich ferngesteuert auf einer im Hallenfußboden eingelassenen Induktionschleife. Die Einsatzbedingung im Rahmen der Farbgebung musste jedoch noch genauer untersucht werden. Im Bereich der Farbgebung könnte es wegen der starken „Farbdampf -Entwicklung" an den Transportwagen zu Störungen kommen. Ferner wäre die Farbgebung im unteren Bereich des Drehautomaten nicht möglich. Daher wird der Drehautomat am Ende des Takts 8 auf ein schienengeführtes Transportsystem umgesetzt. Im Takt 8 erfolgt auch die Montage der Pumpenkombination einschließlich der Qualitätsprüfung aller Komponenten.

Um an den einzelnen Montagen eine Versorgung mit allen Montageteilen sicherzustellen war der Einsatz eines Staplersystems unerlässlich. Durch eine Variantenanalyse konnte ein Flurförderzeug mit einem Hubgerüst, das eine vertikale Lastbewegung ausführen kann, ausgewählt werden.

Nach DIN 18225 und den Arbeitsstätten-Richtlinien müssen in Arbeits- und Lagerräumen über $1000 \mathrm{~m}^{2}$ die Verkehrswege eindeutig gekennzeichnet sein. Es gibt ausschließlich Fußwege, ausschließlich Fahrwege und gemeinsame Fuß- und Fahrwege; außerdem ist zu unterscheiden zwischen Richtungs- und Gegenverkehr. Damit wurde es notwendig im Hallen-Layout diese Wege mit einer Breite von $3 \mathrm{~m}$ in den Grundriss aufzunehmen. Durch die Breite von $3 \mathrm{~m}$ ist es möglich das auch in Spitzenlastzeiten mit Begegnungsverkehr es zu keinerlei Engpässen bei der Versorgung der Montagen kommt.

Wegen des Gewichtes und der Abmessungen der Anbauteile ist die manuelle Montage am Unterkasten nicht möglich. Durch die spezifische Analyse der weiteren Parameter wie Platzbedarf und max. Hebelast wurde ein Drehkran ausgewählt. Drehkrane sind in der Regel ortsfest, können jedoch auch durch Aufsetzen auf ein Fahrwerk (gleisgebunden oder gleislos) bzw. Ponton mobil gemacht werden. Obwohl sie häufig auf Flurniveau angeordnet sind bzw. auf Flurniveau verfahren, werden sie als flurfrei eingestuft, da die Verfahrbewegung der Katze, die Dreh- oder Schwenkbewegung des Auslegers und somit die Förderbewegung für Güter flurfrei und oberhalb der eigentlichen Arbeitsebene ausgeführt wird. Um eine höchstmögliche Verfahrbarkeit des Systems sicherzustellen, muß ein Kransystem mit gleislosem Fahrwerk genutzt werden, so ist es möglich die vom Mitarbeiter in der Produktion benötigten Montageteile immer optimal zum Unterkasten auszurichten und danach zu montieren. Aufgrund der vielen Montageschritte und den daraus resultierenden verschiedenen Montagepunkten ist es notwendig, dass mehrere dieser Systeme einsetzt 


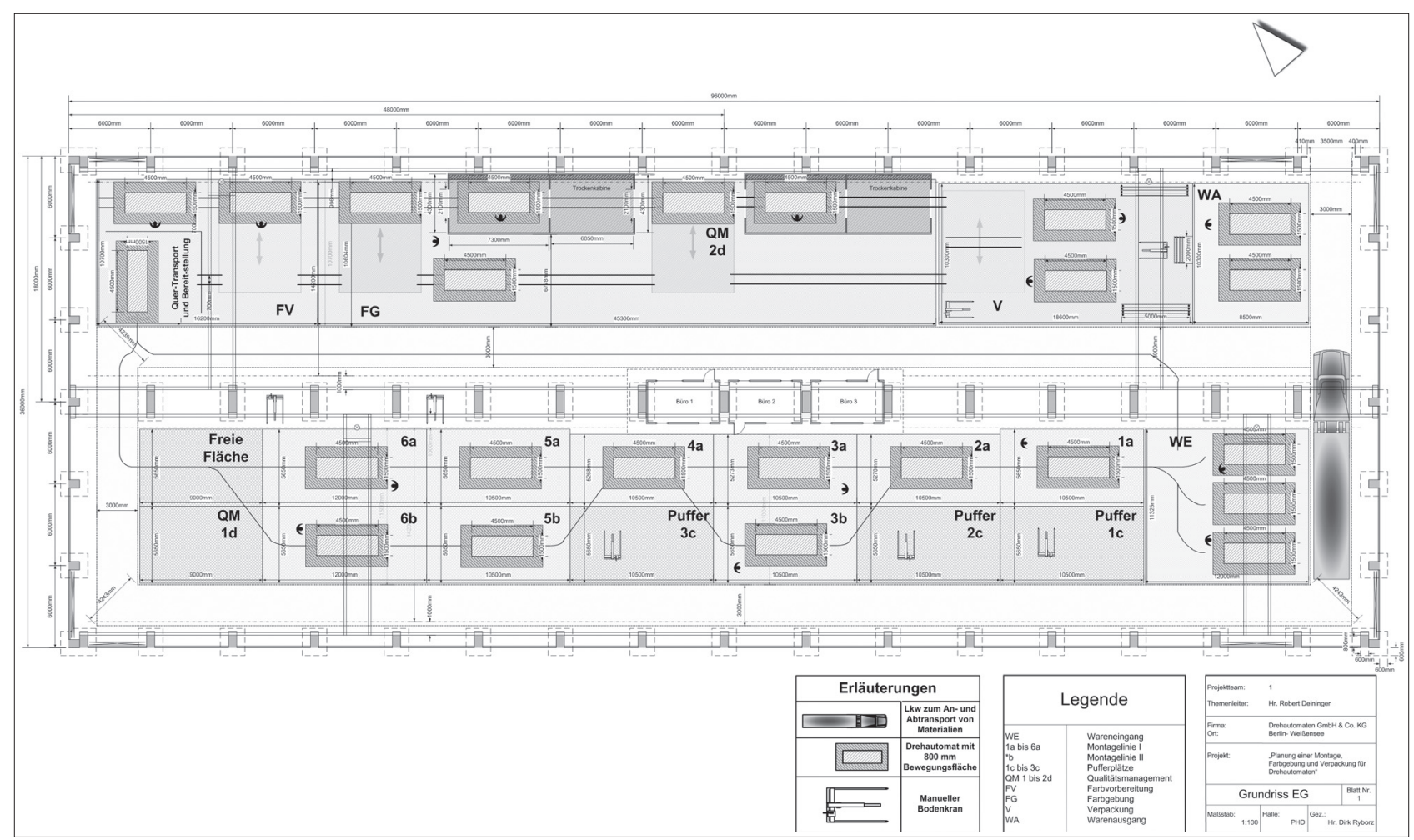

Abb. 3: Layout der Fertigungshalle

werden, um zeitlich optimal abgestimmte Montagelinien zu erzeugen.

Die Farbgebung stellt ein wichtiges Glied der Produktionslinie dar. Im Takt 9 erfolgt die Anlieferung des Drehautomaten in die Farbvorbehandlung, nach dem Umsetzen auf das schienengeführte Transportsystem. Im Takt 10 - Farbvorbehandlung erfolgt das manuelle Abkleben und Spachteln des Drehautomaten. Danach wird dieser über das Transportsystem in die Spritzkabine zur manuellen Farbgebung verbracht. Zu beachten ist, dass in der Spritzkabine besondere Vorgaben zum Explosionsschutz gelten. Nachdem das Spritzen abgeschlossen ist, wird der Drehautomat über das Transportsystem in die Trockenkabine geschleust. Dort wird die erste Farbschicht bei ca. $90^{\circ} \mathrm{C}$ eine Stunde getrocknet. Im Anschluss erfolgt eine Qualitätssichtkontrolle. Sollte ein Fehler festgestellt werden, wird der Drehautomat über ein Verschiebegleis auf eine Ausweichstrecke verbracht. Da in dieser Bearbeitungsphase nur eine Pufferzeit von etwa 60 min zur Verfügung steht, hätte der Qualitätsbeaufrage so auf der Ausweichstrecke die Möglichkeit die Qualitätsprüfung fortzusetzen. Gegebenenfalls wäre es nun möglich, das auf der Ausweichstrecke alle Nacharbeiten erfolgen. Über ein weiteres Verschiebegleis wird der Drehautomat wieder vor die Spritzkabine gebracht und kann in der nächsten Produktionslücke eingesteuert werden. Nachdem nun der Drehautomat die erste Farbgebung erfolgreich durchlaufen hat, beginnt die Einsteuerung in die zweite Farbgebung. Auch nach der zweiten Farbgebung ist eine Trocknung vorgesehen. Danach besteht wiederum die Möglichkeit des Transports über ein Verschiebegleis auf die Ausweichstrecke, um eventuelle Nacharbeiten durchzuführen. Der Drehautomat befindet sich nun am Ende der Fertigungslinie und wird mit Hilfe des Brückenkranes in die Transportverpackung gehoben. Der Wagen des Transportsystems kann nun über die Ausweich- strecke wieder zurückgeführt werden. Das Verpacken des Drehautomaten erfolgt nun manuell in einem speziellen Verpackungsbereich. Dort sind alle Verpackungsmaterialien in einem Pufferlager verfügbar. Beim Verpacken ist die Ladungssicherung in der Transportkiste zu gewährleisten. Die Zolldokumente sind, wenn erforderlich, ebenfalls vom Verpackungsbereich vorzubereiten. Die Zollabnahme wird Stichprobenartig durch das Personal der Zollbehörde durchgeführt. Die Verpackung geschieht unter der Maßgabe, dass die Transportösen an der Maschine genutzt werden können. Dieses Verfahren ist notwendig, um die Transportkiste mit Hilfe eines Krans bewegen zu können. Der Drehautomat kann nach dem Abschluss des Taktes 15 somit in den Versandbereich verbracht werden. Der Versandbereich ist direkt am Haupttor positioniert, dadurch ist ein einfaches Einfahren des Lastkraftwagens zur Übernahme möglich. Nach der Verladung des Gutes durch den Versender erfolgt die Sicherung der Ladung durch den Frachtführer. Mit den zum Einsatz für die Drehautomatenfertigung gewählten technischen Lösungen ergibt sich folgendes Layout für die Fertigungshalle - siehe Abb. 3.

\section{Simulation}

Die Komplexität der Aufgabenstellung stellt im Zusammenspiel mit den gewählten Teillösungen eine schwierig zu überblickende Gesamt-Soll-Konzeption dar. Durch eine genaue Analyse der vorgeschriebenen Rahmenbedingen war die Simulation in Echtzeit unerlässlich. Eine Simulation wird genutzt um komplexe dynamische Systeme analysieren zu können. Die Wirkung der Einflussfaktoren kann so genau untersucht werden, um Erkenntnisse über das reale System zu erhalten. Das Simulationsmodell stellt damit die Gesamtheit aller Abläufe in der Fertigung von 
der Anlieferung bis zum Versand in Echtzeit dar. Die Simulation visualisiert jedes Problem welches innerhalb der Montage auftreten kann. Die verschiedenen Stellgrößen, wie z. B. das Anlieferzeitfenster können so genau betrachtet und optimiert werden.

Zur Simulation wurde das System Simpro 4.1.0 benutzt. Dieses System stellt den Studieninhalt der Lehrveranstaltung „Anwendung logistischer Systeme“ dar. Simpro ist ein universelles Simulationspaket zur Untersuchung des dynamischen Verhaltens komplexer Produktionssysteme. Das so erstellte Modell zeigte die Probleme der Fertigung auf. Im Rahmen des Lösungskonzepts fand eine Bewertung der ermittelten Daten statt. Die Prozesse wurden so genau analysiert und in Teilprozesse zerlegt - siehe Abb. 4. Damit wurde es möglich als ersten Schritt die Schwachstellen innerhalb der einzelnen Montageschritte zu erkennen. Das Hauptproblem, welches sich erstmals in der Simulation darstellte, war die hohe Bearbeitungszeit von ca. 88 h. Durch eine genaue Analyse der einzelnen Takte und der zur Verfügung stehenden Lösungen, wurde die Variante der doppelten Montagen gewählt. Das bedeutet, dass bestimmte Montagetakte nun an zwei Montageplätzen gleichzeitig ablaufen. Der Prozess wurde so parallelisiert. Die Schaffung von zusätzlichen Pufferplätzen an den Montagestationen verkürzt die Montagezeit weiter, da die Bereitstellung nun nicht mehr gleichzeitig mit der Montage geschehen muss.

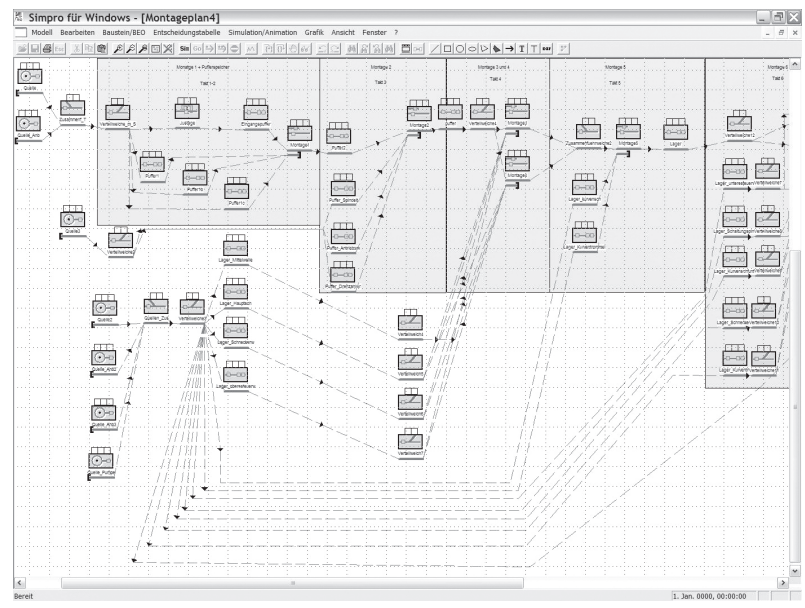

Abb. 4: Simpro Simulation

Durch die so geschaffene Prozessoptimierung wurde die Anzahl der Maschinen im Prozess und damit der Durchsatz erhöht. Im Rahmen einer Potential-Analyse des so geschaffenen Soll-Zustandes wurde ermittelt, dass durch das Ausführen von mehren Montageschritten an einem Montageplatz die Prozessdurchlaufzeit des Drehautomaten verkürzt werden kann. Zum Beispiel wurde so während der Montage der Spindeltrommel und der Laufprüfung schon mit der Montage des Antriebsmotors begonnen.

Der so nochmals optimierte Prozess wurde nun in der Simulation dargestellt und erwies sich als vorteilhaft. Durch eine weitere Anpassung der Montagen wurde ein verbesserter Montagefluss erreicht. Dadurch wurde die Gesamtmontagezeit je Drehautomat und die Durchlaufzeit verkürzt. Dabei wurden alle Anforderungen des Qualitätsmanagementsystems vollständig beibehalten. Darüber hinaus wurden zusätzliche Stellflächen geschaffen, um die Durchführung von weiteren Qualitätsprüfungen sicherzu- stellen. Um nun die ermittelten Parameter im Kontext mit den physischen Grenzen der Halle betrachten zu können, wurde das Simulationsmodell in die Produktionshalle gesetzt. Dadurch wurde es möglich in den engen Grenzen der Halle die Auslastung der Transportwege sowie die Funktion des fahrerlosen Transportsystems zu untersuchen. Als Schwachstelle wurde das Anlieferkonzept erkannt. Mit den so erhaltenen Informationen wurde eine Analyse erstellt, die zum Ziel hatte, eine neue Strategie zur Belieferung zu entwickeln. Da es während des Produktionsprozesses zu einem permanenten Bedarf an Montageteilen kommt, ist es nicht möglich diese per Just-in-Time anzuliefern. Durch die Belegungszeit des Brückenkranes im Hallenabschnitt der Belieferung musste das Anlieferkonzept verändert werden. Nach eingehender Prüfung der Möglichkeiten wurde die Variante der Schaffung eines Zwischenlagers außerhalb der Produktionshalle als optimale Lösung ermittelt. Dadurch ist es möglich die Belieferung der einzelnen Montageplätze individuell zu bestimmen und so größtmögliche Flexibilität zu gewährleisten. Die jetzt geschaffene Soll-Konzeption stellt damit einen hohen Grad der Zielerreichung dar - siehe Abb. 5.

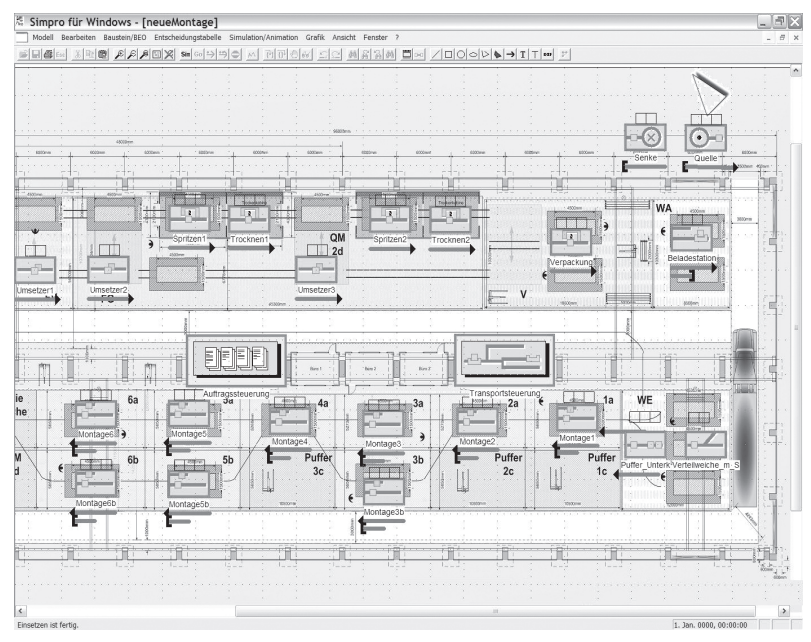

Abb. 5: Simpro Simulation im Hallenlayout

\section{Pädagogischer Effekt}

Elementare Bestandteile im Studienschwerpunkt Unternehmenslogistik an der TFH Wildau sind die Vermittlung von Kenntnissen über Strukturen, Strategien und Systemen zur Lösung logistischer Aufgaben. Eine hohe Bedeutung bei der Vermittlung dieses Wissens, hat die Einbindung von konkreten Fallbeispielen aus der Industrie innerhalb der Lehrveranstaltungen. Zur Umsetzung dieser Ziele und Methoden wurde das Studienfach Produktionslogistik ganzheitlich auf die Erarbeitung eines logistischen Gesamtkonzeptes am Beispiel der Fertigung von Werkzeugmaschinen ausgerichtet. Für die Studenten ergabt sich daraus eine sehr praxisnahe Aufgabenstellung, die zahlreiche theoretische Kenntnisse aus dem Grundstudium abfordert. Zum einen waren fundierte Kenntnisse im Projektmanagement notwendig, um Anforderungen zur Teamorganisation, sowie zur Zeit- und Ressourcenplanung zu bewältigen. Zum anderen wurde durch die Bearbeitung des Projektes die Betrachtung von komplexen 
Systemen weiter geschult. Die vorlesungsübergreifende Projektarbeit förderte das vernetzte Denken und die Anwendung unterschiedlicher Lehrinhalte des Studiums. Durch die Problemstellung in den einzelnen Bereichen war es notwendig die unterschiedlichen Varianten der Lösungsmöglichkeiten und die damit verbunden technischen Umsetzungen zu untersuchen. Diese Projektarbeit setzte die Anwendung eines breiten Spektrums an Wissen, sowie ein sehr gutes technisches Verständnis bei den Teammitgliedern voraus. So war es notwendig Probleme der Organisationsentwicklung, der Planung von logistischen Systemen und der Analyse technischer Lösungen zu verknüpfen. Dieses Projekt setzte einen hohen Anteil an Eigeninitiative voraus, um die komplexen Problemstellungen außerhalb der Hochschule zu untersuchen. Durch die Einbeziehung eines Austauschstudenten wurde die Projektarbeit positiv beeinflusst. Durch die damit eingebrachte „andere Sicht“, wurde die Projektarbeit, insbesondere die Problemdiskussion zusätzlich bereichert. Die deutschen Studenten konnten so für das Arbeiten an einem gemeinsamen Projekt mit ausländischen Studenten ein hohes Maß an Erfahrungswerten gewinnen. Die Förderung der Teamarbeit und die damit verbundene Arbeitsteilung ermöglichte jedem Teammitglied auf einem speziellen Gebiet Kernkompetenzen zu entwickeln und dadurch das Team individuell zu prägen. Bei auftretenden Problemen und Fragen war es dem Team jederzeit möglich den betreuenden Dozenten des Projektes Herrn Prof. Dr. -Ing. habil. Bernd Hentschel zu konsultieren. Den Studenten zeigte die eigenständige Ausarbeitung des umfangreichen Gesamtprojektes die Probleme und Herausforderungen bei der Planung von produktionslogistischen Systemen, die in einer herkömmlichen Vorlesung nur schwer zu vermitteln wären.

\section{Zusammenfassung}

Dem Projektteam war die Aufgabe gestellt das technologische und logistische Konzept für die Produktion von Drehautomaten in einer vorhandenen Halle bei vorgegeben Arbeitstakten zu erarbeiten.

Durch die Variantenanalyse der verschiedenen technologischen Möglichkeiten bezüglich der maßgeblichen Kenngrößen wie Platzbedarf, Rüstzeit, Standzeit und Transportgeschwindigkeit wurden die Technologien ausgewählt und dass Hallenlayout für die konzipierte Fließfertigung mittels Microsoft Visio erstellt. Die entwickelte Fertigung besteht aus 6 Montage - und 4 Pufferplätzen. In der Farbvorbereitung bestehen zwei Arbeitsplätze sowie ein Bereitstellungsplatz. Im Bereich der Farbgebung wurden zur Vermeidung eines Produktrücktransports nach der Farbvorbereitung jeweils eine Spritz- und Trockenkabine hintereinander angeordnet. Im Bereich der Verpackung sind zwei Arbeits- und zwei Versandplätze vorgesehen. Dies ermöglicht auch während Spitzenlastzeiten eine problemlose Bearbeitung.

Mit dem erarbeiteten Soll-Konzept wurde die Zielstellung der Lehrveranstaltung erreicht und durch die Anwendung einer logistischen Simulationssoftware eine komplexere Problembearbeitung durchgeführt.

\section{Anmerkungen}

Dank gilt dem wissenschaftlichen Mitarbeiter, Herrn Dipl.Ing. Peter Wasser, der dem Projektteam bei der Erstellung der Simulation Unterstützung gewährte.

\section{Teammitglieder}

Robert Deininger

Technische Fachhochschule Wildau

Fachbereich Ingenieurwesen/Wirtschaftsingenieurwesen

- Logistik

rdeining@igw.tfh-wildau.de

\section{Sebastian Forkert}

Technische Fachhochschule Wildau

Fachbereich Ingenieurwesen/Wirtschaftsingenieurwesen

- Logistik

sforkert@igw.tfh-wildau.de

\section{Dirk Ryborz}

Technische Fachhochschule Wildau

Fachbereich Ingenieurwesen/Wirtschaftsingenieurwesen

- Logistik

dryoborz@igw.tfh-wildau.de

\section{Silvana Adolph}

Technische Fachhochschule Wildau

Fachbereich Ingenieurwesen/Wirtschaftsingenieurwesen

- Logistik

sadolph@igw.tfh-wildau.de

\section{Jan-Jaap van Wikselaar}

Hogeschool van Arnehm en Nijmegen

Hoger Economisch en Administratief Onderwijs

- European Logistics

jjwikselaa@student.han.nl

\author{
Autor \\ Robert Deininger \\ Technische Fachhochschule Wildau \\ Fachbereich Ingenieurwesen/Wirtschaftsingenieurwesen \\ - Logistik \\ rdeining@igw.tfh-wildau.de
}

